\title{
Off-axis Electron And Light Optical Holograms: How They Process And How They Compare
}

\author{
E. Voelkl,* W.R. Usry,* B.G. Frost** \\ * nLine Corp., 4150 Freidrich Lane, Suite A, Austin, TX 78744 \\ ** EM Facility, University of Tennessee, Knoxville, TN 37996
}

It has taken more than half a century to develop the idea of Gabor for holography [1] into a true technological application. For this event, both light optics and electron optics have played various, important roles to mature this technology. Today, the necessary secondary technologies are in place to transform holography to an everyday tool e.g., for the semiconductor industry.

Originally, holography was invented to solve an electron optical problem: It was known from the work of O. Scherzer that (the spherical) aberration of the objective lens could not be compensated as in light optics with an optical element of 'opposite' aberrations [2]. Such a limitation was a serious handicap for the development of electron microscopy because of the resulting limitation in resolution. That was when Gabor proposed a way out of the dilemma: the idea to correct for the aberrations after the image was already taken by recording a hologram [1].

After the birth of the idea, its technological limitations were visible very quickly. The first publications in electron holography show holograms that were limited to about 10 interferences fringes by the lateral coherence of the electron beam [3]. As light optics offered coherent sources, it comes at no surprise that holography became actively developed and refined in light optics and other fields. Due to the necessary processing of holograms, either on the optical bench or in the computer, holography could not fully develop: it remained basically a static technology.

For electron microscopy however, the basic problem of (spherical) aberration was still there. In the 70's electron sources had been discovered that provided a significantly improved coherent beam and thus reopened the door for electron holography [4]. Still, holograms were recorded on film and processed afterwards - an excruciatingly slow process. Yet in 1986, it was demonstrated that the task of spherical aberration correction could, in principle, be accomplished as initially intended by Gabor [5]. This demonstration together with the development of digital cameras (CCDs) and the ever-increasing computational processing speed very quickly drove the development first in electron holography and then in light holography.

Electrons and photons are very different particles - or waves. The parallel development in electron and light optics is due to the fact that the equations describing them appear similar if the time dependence is dropped. Electrons are described by the Schroedinger equation (for one electron)

$$
\left(-h^{2} / 8 \pi^{2} m\right) \Delta \psi(\boldsymbol{r}, t)=\mathrm{i} h / 2 \pi \delta_{t} \psi(\boldsymbol{r}, t) \text { or time independent: }\left(-h^{2} / 8 \pi^{2} m\right) \Delta \psi(\boldsymbol{r})=\mathrm{E} \psi(\boldsymbol{r})
$$

whereas photons (for an arbitrary number) are described by the wave equation

$$
\mathrm{c}^{2} \Delta \psi(\boldsymbol{r}, t)=\delta_{t t} \psi(\boldsymbol{r}, t) \quad \text { or time independent: } \quad-\mathrm{c}^{2} \Delta \psi(\boldsymbol{r})=\omega^{2} \psi(\boldsymbol{r})
$$


with $h$ as the Plank constant, $\delta_{t t} \psi$ describing the second derivative of the wave function $\psi$ with time and $\Delta$ the Laplace operator. In the time independent form both equations are similar despite the fact that the waves have different meanings.

The nature of light, i.e., photons belonging to the particle class of Bosons, has brought about the availability of laser light for light optics. For electrons however, being Fermions, an electron laser is not possible. Therefore, the performance of light holography should outperform electron holography by far due to the improved coherence conditions from laser light. However as will be shown, both fields have their advantages and disadvantages.

An overview will be given of the developments and applications of both electron and light holography. Whereas in electron holography the transmission mode will be reviewed, for light holography the reflective mode will be discussed and parallels being drawn (FIG 1 and FIG 2). Last but not least, highlights will be shown in the development of a holographic tool for defect detection for the semiconductor industry developed at ORNL and nLine Corporation [6].

\section{References}

[1] D. Gabor, Nature 161(1948), 777-778

[2] O. Scherzer, Z. Phys. 101, 593 (1936)

[3] Introduction to Electron Holography, Eds., E Voelkl et al., Plenum Press 1998

[4] D.W. Tuggle, JZ Li, LW Swanson, J. Microscopy, 140, 293 (1985)

[5] F.J. Franke et al, Proc. XI ${ }^{\text {th }}$ Int. Cong. On Electron Microscopy, Kyoto, 1986, 677-678.

[6] C.E. Thomas et al., Proc. SPIE Vol. 4692, 180-194

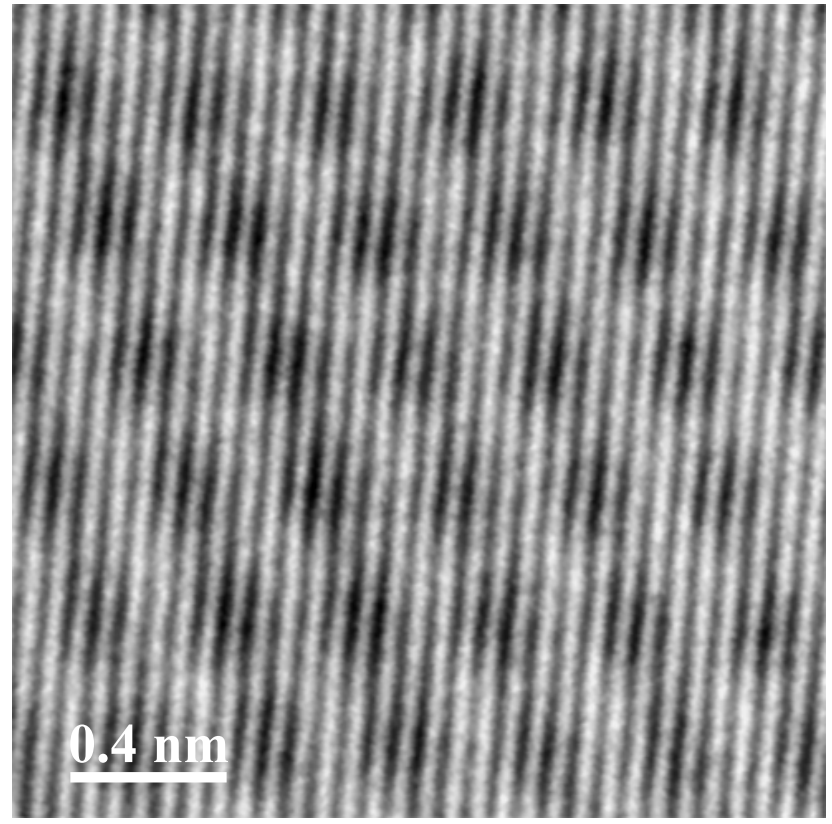

FIG. 1. Detail of an electron hologram of a $\mathrm{CeO}_{2}$ crystal in $<110>$ direction. The visible fine grain in the image indicates that the hologram was originally recorded on film.

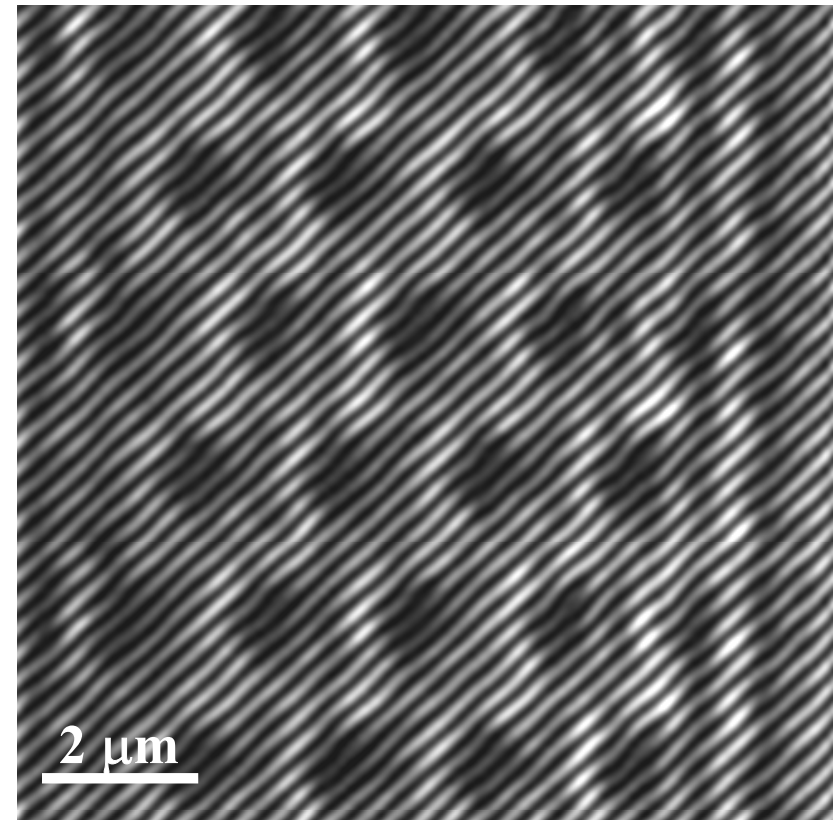

FIG. 2. Detail of a light optical hologram of a semiconductor structure in reflection mode. The hologram was recorded digitally on a $2 \mathrm{k} \times 2 \mathrm{k}$ CCD camera that can handle up to $30 \mathrm{fps}$. 